\title{
PARÂMETROS PARA MONITORAMENTO DA COMPOSTAGEM DE CARCAÇAS DE FRANGO
}

\author{
Ed Carlo Rosa Paiva ${ }^{1}$, Antonio Teixeira Matos², Renata Tâmara Pereira de Barros ${ }^{3}$, Tatiana Dias Ribeiro da Costa ${ }^{4}$,
} Antover Panazzolo Sarmento 5

\begin{abstract}
RESUMO
O objetivo deste trabalho foi verificar o uso da relação entre Carbono e Nitrogênio $(\mathrm{C} / \mathrm{N})$ e do índice definido pela relação entre a Capacidade de Troca Catiônica e o Carbono Orgânico Total (CTC/COT) para o monitoramento da compostagem de carcaças de frango. O experimento consistiu na compostagem de carcaças de frango pelos métodos: composteira e leira estática aerada (LEA). Os materiais utilizados na compostagem foram: cama-de-frango, bagaço de cana-de-açúcar, palha de café e carcaça de frango. A proporção dos materiais utilizados na montagem dos diferentes tratamentos foi de $0,3 \mathrm{~kg}$ de bagaço de cana-de-açúcar ou palha de café para cada $1 \mathrm{~kg}$ de carcaça de frango e 2 $\mathrm{kg}$ de cama-de-frango. Uma composteira foi construída com uma base de $1,5 \mathrm{~m}^{2}$ e altura útil de $1,5 \mathrm{~m}$ e as LEAs com, aproximadamente, $3 \mathrm{~m}$ de comprimento, $2 \mathrm{~m}$ de largura e $1 \mathrm{~m}$ de altura. Diante dos resultados obtidos, podese concluir que a relação $\mathrm{C} / \mathrm{N}$ não se mostrou adequada para descrever a evolução da compostagem desse tipo de resíduo, especialmente no final da fase de degradação ativa e na fase de humificação. O índice CTC/COT se mostrou mais adequado para descrever o processo de compostagem. A CTC descreveu a redução do tamanho das partículas e, consequentemente, o aumento da superfície específica, além de estar relacionada ao processo de humificação. O COT descreveu a presença de matéria orgânica e a sua redução indicou a redução da matéria orgânica ou a mineralização do material orgânico.
\end{abstract}

Palavras-chave: composto, estabilidade, maturidade.

\section{ABSTRACT \\ PARAMETERS FOR MONITORING OF POULTRY CARCASS COMPOSTING}

The aim of this study was to investigate the use of the relationship between carbon and nitrogen $(\mathrm{C} / \mathrm{N})$ and the index defined by the relationship between the Cation Exchange Capacity and Total Organic Carbon (CTC/COT) for monitoring the composting of poultry carcasses. The experiment consisted of composting poultry carcasses by the methods: composting windrow and aerated static piles (ASP). The materials used in composting were poultry litter, sugarcane bagasse, coffee straw and poultry carcass. The proportion of the materials used in the assembly of the different treatments was $0.3 \mathrm{~kg}$ of sugarcane bagasse or coffee straw for every $1 \mathrm{~kg}$ of poultry carcass and $2 \mathrm{~kg}$ of poultry litter. A compost bin with a base $1.5 \mathrm{~m}^{2}$ and working height of $1.5 \mathrm{~m}$, and the ASPs were approximately $3 \mathrm{~m}$ long, $2 \mathrm{~m}$ wide and $1 \mathrm{~m}$ high. Based on these results it can be concluded that the $\mathrm{C} / \mathrm{N}$ ratio was not adequate to describe the evolution of this type of waste composting, especially in the late phase of active degradation and the humification stage. The CEC/TOC index was more appropriate to describe the composting process. The CEC described the reduction of particle size and resultant increase in the specific surface, and is also related to the humification process. The TOC described the presence of organic matter and its reduction indicated a reduction in organic matter or organic matter mineralization.

Keywords: compost, stability, maturity

\section{Recebido para publicação em 28/07/2014. Aprovado em 13/10/2015.}

1 - Engenheiro Civil, D.S. em Eng. Agrícola. Professor Adjunto FENG/RC-UFG mail: edcarlopaiva@yahoo.com.br.

2 - Engenheiro Agrícola, D.S. em Solos e Nutrição de Plantas; Professor Titular DESA/UFMG. E-mail: atmatos@desa.ufmg.br.

3 - Enga Agrícola e Ambiental. Mestranda em Ciência e Tecnologia da Sustentabilidade da Univ. Fed. de São Paulo. natufv2004@yahoo.com.br

4 - Engenheira Agrícola e Ambiental da UFV. tatianadias749@hotmail.com

5 - Engenheiro Agrícola, D.S. em Engenharia Agrícola; Professor Assistente FENG/RC-UFG. E-mail: antoverps@gmail.br. 


\section{INTRODUÇÃO}

Uma das técnicas mais difundidas em todo mundo para o tratamento e disposição de resíduos orgânicos é a compostagem. Essa técnica apresenta uma série de variantes, tais como leiras reviradas manualmente ou mecanicamente, leiras estáticas, reatores fechados mecanizados e composteiras, utilizadas no tratamento dos mais diferentes tipos de resíduos orgânicos. A técnica da compostagem, sendo bem conduzida, produz como produto final um material estabilizado quimicamente e com reduzido número de microrganismos patogênicos. Tal produto, denominado composto, caracterizase por ser um material humificado e excelente condicionador do solo (PAIVA, 2014).

Estudos sobre compostagem em leiras têm sido desenvolvidos para diferentes tipos de resíduos, como bagaço de cana-de-açúcar, capim Napier picado, palha de café, aparas de madeira e resíduo de suinocultura utilizando reviramento (MATOS et al., 1998; ROS et al., 2006; CHIUMENTI et al., 2007), lodos de águas residuárias e a fração orgânica dos resíduos sólidos urbanos; utilizando leiras estáticas aeradas (GUARDIA et al., 2008; WANG et al., 2011).

A compostagem de carcaças de aves, entretanto, tem sido desenvolvida, basicamente, pelo método da composteira, em geral, seguido do processo de leiras por reviramento manual (KUMAR et al., 2007; ORRICO JÚNIOR et al., 2010; PAIVA et al., 2012). Segundo Graves et al. (2000), a compostagem de carcaças em composteira é a técnica de tratamento de custo e tecnologia mais acessível aos produtores de aves. Entretanto, o processo por leiras estáticas aeradas (LEAs) tem sido um dos mais difundidos para o tratamento de resíduos orgânicos por apresentar custos de operação similares ao processo Windrow ou em leiras por reviramento, além de ser o único processo

Quadro 1. Critérios atualmente utilizados para caracterizar a qualidade do composto orgânico

\begin{tabular}{|c|c|c|}
\hline Tipo & Parâmetros & Análises \\
\hline \multirow{5}{*}{ Físico: } & \multirow{5}{*}{$\begin{array}{l}\text { Odor } \\
\text { Cor } \\
\text { Temperatura } \\
\text { Tamanho de partículas e } \\
\text { material inerte }\end{array}$} & Verificação olfativa \\
\hline & & Visual \\
\hline & & Por meio de sondas conectadas a termômetros \\
\hline & & Uso de peneiras ou mesmo visuais \\
\hline & & Determinação de sólidos fixos com a utilização de mufla \\
\hline \multirow{7}{*}{ Químicos: } & Carbono e nitrogênio & $\mathrm{C} / \mathrm{N}$ em extrato sólido e líquido \\
\hline & CTC & CTC e relação CTC/COT \\
\hline & Extrato aquoso & $\mathrm{pH}, \mathrm{CE}, \mathrm{CO}$ e íons \\
\hline & Nitrogênio mineral & $\mathrm{N}-\mathrm{NH}_{4}$ e N-NH $\mathrm{N}_{4} / \mathrm{N}^{-\mathrm{NO}_{3}}$ \\
\hline & Poluentes & Metais pesados e orgânicos \\
\hline & $\begin{array}{l}\text { Qualidade da matéria } \\
\text { orgânica }\end{array}$ & $\begin{array}{l}\text { Composição orgânica: lignina, carboidratos complexos, lipídios e } \\
\text { açucares }\end{array}$ \\
\hline & Humificação & $\begin{array}{l}\text { Caracterização de substâncias húmicas: análise de grupos funcionais } \\
\text { e elementares, distribuição do peso molecular, relação E4/E6 entre } \\
\text { outras }\end{array}$ \\
\hline \multirow{3}{*}{ Biológicos: } & $\begin{array}{l}\text { Indicadores de atividade } \\
\text { microbiológica }\end{array}$ & $\begin{array}{l}\text { Respiração (consumo de } \mathrm{O}_{2} \text {, produção de } \mathrm{CO}_{2} \text { e biodegradabilidade), } \\
\text { atividade enzimática e biomassa microbiana }\end{array}$ \\
\hline & Fitotoxicidade & Testes de germinação e crescimento de plantas \\
\hline & Outros & Sementes daninhas viáveis, patógenos e testes de ecotoxicidade \\
\hline
\end{tabular}

Fonte: Adaptado de Bernal et al. (2009)

Notas: CTC: capacidade de troca catiônica, CTC/COT: relação capacidade de troca de cátions por carbono orgânico total, CE: condutividade elétrica, $\mathrm{CO}$ : Carbono orgânico, $\mathrm{C} / \mathrm{N}$ : relação carbono por nitrogênio, $\mathrm{N}_{-} \mathrm{NH}_{4}$ : nitrogênio na forma de amônio, $\mathrm{N}-\mathrm{NO}_{3}$ : nitrogênio na forma de nitrato. 
que combina adequada eliminação de patógenos com alto grau de estabilização do material orgânico (GOLUEKE et al., 1980). Devido a essas características, acredita-se que a compostagem de carcaças possa ser uma excelente alternativa à crescente demanda de tratamento e disposição final dos resíduos gerados na produção de carnes de frango no Brasil.

São diversos os critérios que podem ser utilizados para caracterização da qualidade do composto, no Quadro 1 está exposto um resumo destes.

Para avaliar o desenvolvimento do processo de compostagem a variável mais utilizada tem sido a relação $\mathrm{C} / \mathrm{N}$, ou seja, a proporção entre os nutrientes carbono e nitrogênio na massa em compostagem. Porém, estudos têm mostrado que essa variável não tem apresentado bons resultados para a compostagem de resíduos com elevada concentração de nitrogênio e, portanto, baixa relação $\mathrm{C} / \mathrm{N}$, como é o caso dos resíduos de carcaças de animais e de esterco de galinha (DORES-SILVA et al., 2011; VALENTE et al., 2011; MONACO et al., 2013).

Conforme exposto por Roig et al. (1988), Jiménez e Garcia (1992) e Melo et al. (2008), começa a ser utilizado como um melhor índice para verificação do estádio de humificação do material a relação CTC/COT. Nesses trabalhos, citam-se que os valores limites para se considerar compostos bem humificados estão acima de $1,7 \mathrm{~mol}_{\mathrm{c}} \mathrm{kg}^{-1}$ para estercos, 1,9 mol $_{\mathrm{c}} \mathrm{kg}^{-1}$ para composto de lixo e 1,7 mol $_{\mathrm{c}} \mathrm{kg}^{-1}$ para lodo de esgoto.

Sendo assim, o objetivo deste trabalho foi analisar a adequação da relação $\mathrm{C} / \mathrm{N}$ e do índice CTC/COT como parâmetros de monitoramento da compostagem de carcaças de frango.

\section{MATERIAL E MÉTODOS}

$\mathrm{O}$ experimento baseou-se na compostagem de carcaças de frango pelos métodos da composteira e leiras estáticas aeradas (LEAs). Realizou-se o experimento nas dependências do Departamento de Engenharia Agrícola (DEA) da Universidade Federal de Viçosa (UFV). Os materiais utilizados na compostagem foram; cama de frango, bagaço de cana-de-açúcar, palha de café e carcaça de frango.

As proporções dos materiais (cama de frango, bagaço de cana-de-açúcar, carcaça de frango e água) utilizados na montagem dos diferentes tratamentos seguiram as recomendações de Dam et al. (2009), tendo sido utilizados $0,3 \mathrm{~kg}$ de bagaço de cana-de-açúcar ou palha de café para cada $1 \mathrm{~kg}$ de carcaça de frango e $2 \mathrm{~kg}$ de cama-de-frango. Tais proporções visam manter uma adequada relação $\mathrm{C} / \mathrm{N}$ na massa do material em compostagem, que para carcaças de animais está entre 10 e 20/1.

No Quadro 2 estão apresentados os experimentos montados e monitorados, bem com a composição de cada um deles.

A composteira foi construída com $1,5 \mathrm{~m}$ de base, $1,5 \mathrm{~m}$ de comprimento e $1,5 \mathrm{~m}$ de altura, as leiras foram montadas com seção transversal trapezoidal (LEA 01), com medidas aproximadas de $2 \mathrm{~m}$ de base maior, 1, $5 \mathrm{~m}$ de base menor e 1 metro de altura e triangular (LEA 02 e 03 ), com medidas aproximadas de $2 \mathrm{~m}$ de base e 1 metro de altura, tendo todas o comprimento de $3 \mathrm{~m}$. A composteira e a LEA 01, montadas com carcaças de frango inteiras, foram montadas de forma estratificada segundo recomendações de Dam et al. (2009).

Quadro 2. Experimentos monitorados.

\begin{tabular}{ll}
\hline \multicolumn{1}{c}{ Experimento } & \multicolumn{1}{c}{ Descrição } \\
\hline Composteira (COM) & Carcaças de frango inteiras, palha de café e cama de frango; \\
\hline LEA 01 (L01) & Carcaças de frango inteiras, bagaço de cana-de-açúcar e cama de frango; \\
\hline LEA 02 (L02) & Carcaças de frango trituradas, bagaço de cana-de-açúcar e cama de frango; \\
\hline LEA 03 (L03) & Carcaças de frango trituradas, palha de café e cama de frango.
\end{tabular}


A montagem da composteira iniciou com a colocação de uma camada de $45 \mathrm{~cm}$ de cama de frango seca no fundo seguida de uma camada de $15 \mathrm{~cm}$ de palha de café. Depois disso, adicionou-se uma camada de carcaças, cobrindo, em seguida, com cama de frango até haver a formação de uma camada de, pelo menos, $5 \mathrm{~cm}$ de material sobre as carcaças. Adicionou-se água (o equivalente a $20 \%$ da massa das carcaças) sobre o material para umedecê-lo. Repetiu-se essa sequência até ser colocada a última camada de aves. Cobriu-se, então, a pilha de material com uma camada dupla de cama de frango seca. Para LEA 01 obedeceu-se a mesma sequência, porém com a cama de frango e o bagaço de cana misturado antes da colocação no fundo, entre as carcaças e cobertura final. Nas LEAs 02 e 03, que utilizaram carcaças frango trituradas, antes da montagem, os resíduos foram pré-misturados buscando a sua homogeneização, segundo recomendações de Pereira Neto (2007).

As LEAs continham cada uma um ventilador centrífugo (motor elétrico de $1 / 4 \mathrm{HP}$ de potência) de funcionamento controlado por timer analógico (precisão de 15 minutos) e termostato digital (precisão de $0,1^{\circ} \mathrm{C}$ ).

As temperaturas foram medidas diariamente em três pontos da massa (base, centro e topo). A relação $\mathrm{C} / \mathrm{N}$ e $\mathrm{COT}$ foram determinadas a cada 15 dias (nos primeiros 60 dias) nas LEAs e a cada 20 dias (nos primeiros 60 dias) na composteira. A CTC e o índice CTC/COT foram determinados a partir do $30^{\circ}$ dia de experimento. Na condução dos experimentos em LEAs, buscou-se manter o conteúdo de água na faixa 45 e $55 \mathrm{dag}^{-1}$, considerada ideal, segundo Kiehl (1985) e Pereira Neto (2007), para rápida degradação do material orgânico.

No Quadro 3 estão apresentadas as variáveis estudadas, bem como os métodos utilizados na sua determinação.

Para consideração do fim da fase ativa, bem como da fase de maturação, foram seguidas as recomendações apresentadas por Kiehl (1985). Assim, o fim da fase ativa de degradação foi considerado alcançado quando a relação $\mathrm{C} / \mathrm{N}$ do material passou a ser menor que 18/1 e, ou, a temperatura das pilhas menor que $40{ }^{\circ} \mathrm{C}$. Findada a fase ativa, o material das leiras e da composteira foram transportados para pátio para que ocorresse a fase de maturação. A fase de maturação foi considerada findada quando a relação $\mathrm{C} / \mathrm{N}$ no material ficou em torno de 10/1, valor indicativo de estabilização do material, conforme sugerido por Kiehl (1985).

Quadro 3. Variáveis estudadas e respectivos métodos de determinação.

\begin{tabular}{|c|c|c|c|}
\hline Variável & Unidade & Tipo & Observação \\
\hline Temperatura da leira & ${ }^{\circ} \mathrm{C}$ & Medida & Medidas "in loco".-* \\
\hline Carbono orgânico total (COT) & dag $\mathrm{kg}^{-1}$ & Determinada & $\begin{array}{l}\text { Determinada segundo o método da } \\
\text { combustão (KIEHL, 1985) }\end{array}$ \\
\hline Nitrogênio $(\mathrm{N})$ & dag $\mathrm{kg}^{-1}$ & Determinada & $\begin{array}{l}\text { Nitrogênio total determinado segundo } \\
\text { método Kjedahl (APHA et al., 2012) }\end{array}$ \\
\hline Relação C/N & Adimensional & Calculada & $\mathrm{C} / \mathrm{N}$ \\
\hline Conteúdo de água & dag $\mathrm{kg}^{-1}$ & Determinada & Método da estufa (APHA et al., 2012) \\
\hline Sólidos Voláteis (SV) & dag $\mathrm{kg}^{-1}$ & Calculado & $\begin{array}{l}\text { COT }=\text { SV/1,8 (base seca) (RICHARD, } \\
\text { 1996; BORDER, 2002) }\end{array}$ \\
\hline $\begin{array}{l}\text { Capacidade de Troca Catiônica } \\
\text { (CTC) }\end{array}$ & $\mathrm{cmol}_{\mathrm{c}} \mathrm{kg}^{-1}$ & Determinado & $\begin{array}{l}\text { Método proposto por HARADA e INOKO } \\
(1980)\end{array}$ \\
\hline Índice CTC/COT & Adimensional & Calculado & CTC / COT \\
\hline
\end{tabular}

*Medidas em três pontos da leira (base, centro e topo) com o uso de sondas termopar e termômetro digital. 


\section{RESULTADOS E DISCUSSÃO}

No Quadro 4 estão apresentados os valores obtidos para as variáveis e relação $\mathrm{C} / \mathrm{N}$ determinadas durante o período de compostagem do material.

Como pode ser observada a relação $\mathrm{C} / \mathrm{N}$ de todos os tratamentos, após 60 dias de compostagem, apresentaram valores superiores aos iniciais, ou seja, para os dias 0 e 60 foram obtidos os seguintes valores: composteira $(19,2,27,6)$, LEA $01(13,8$, 17,9), LEA $02(13,8,15,1)$ e LEA $01(13,6,17,8)$, respectivamente. Esse comportamento tem sido observado também por outros autores, Kumar et al. (2007), trabalhando com composteira, observaram aumento no valor da relação $\mathrm{C} / \mathrm{N}$ no composto final, em comparação com o obtido inicialmente. Esses autores obtiveram valores finais de relação $\mathrm{C} / \mathrm{N}$ de $15,9( \pm 0,76)$ a $23,19( \pm 4,62)$ no tratamento com palha de arroz e de $16,35( \pm 1,06)$ a 18,0 $( \pm 1,64)$ no tratamento com feno de sorgo. Flynn e Wood (1996), analisando as mudanças químicas e de temperaturas na compostagem de cama de frango, também observaram que em leiras que iniciaram com relação $\mathrm{C} / \mathrm{N}$ de 24,26 e 26:1, após 84 dias de compostagem, as relações $\mathrm{C} / \mathrm{N}$ foram aumentadas ou ligeiramente reduzidas para valores de 22; 27 e 28:1, respectivamente. Isso pode estar relacionado às altas concentrações de nitrogênio e baixas relações $\mathrm{C} / \mathrm{N}$, presentes, inicialmente, no material a ser compostado, seguidas de perda por volatilização da amônia ao longo do processo.

Tal comportamento inviabilizou a utilização da metodologia proposta por Kiehl (1985), que recomendava que o fim da fase ativa de degradação seria alcançado quando a relação $\mathrm{C} / \mathrm{N}$ do material passasse a ser menor que 18/1 e a fase de maturação seria considerada findada quando a relação $\mathrm{C} / \mathrm{N}$ no material ficasse em torno de 10/1. Acredita-se que tal recomendação se adeque mais para a compostagem de resíduos orgânicos convencionais, nos quais não há a presença de restos de animais, e cujo processo se inicia com relação $\mathrm{C} / \mathrm{N}$ em torno de 30/1. Nessas

Quadro 4. Relação C/N e COT dos materiais compostados.

\begin{tabular}{cccccc}
\hline Experimentos & Dias & COM & L01 & L02 & L03 \\
\hline & 0 & 19,2 & 13,8 & 13,8 & 13,6 \\
& 15 & - & 5,8 & 9,0 & 8,4 \\
C/N & 20 & 9,6 & - & - & - \\
& 30 & - & 8,9 & 8,9 & 7,5 \\
& 40 & 13,3 & - & - & - \\
& 45 & - & 9,3 & 8,4 & 8,0 \\
& 60 & 27,6 & 17,9 & 15,1 & 17,8 \\
& 90 & 22,7 & 18,1 & 14,6 & 17,4 \\
\hline & 0 & 46,0 & 47,8 & 47,8 & 47,8 \\
COT (dag kg-1) & 15 & - & 46,0 & 41,9 & 45,0 \\
& 20 & 45,5 & - & - & - \\
& 30 & - & 41,5 & 37,7 & 40,5 \\
& 40 & 46,5 & - & - & - \\
& 45 & - & 36,4 & 37,4 & 34,0
\end{tabular}


circunstâncias a relação $\mathrm{C} / \mathrm{N}$ não se mostrou um bom parâmetro de avaliação do processo, tal como previsto inicialmente na metodologia.

Quanto à CTC e ao índice CTC/COT, pode ser observado no Quadro 5 que ambos apresentaram tendência de aumento à medida que o processo de degradação do material orgânico ocorria, conforme esperado, uma vez que tais variáveis estão relacionadas com o grau de humificação ou maturação do composto.

A CTC, após 60 dias de manutenção do material na composteira, foi de $175 \mathrm{cmol}_{\mathrm{c}} \mathrm{kg}^{-1}$, o COT foi 41,5 dag $\mathrm{kg}^{-1}$ e a relação CTC/COT foi de 4,2. Após 90 dias de compostagem, 30 dias de reviramento no pátio de compostagem, o material retirado da composteira apresentou CTC de 209 $\mathrm{cmol}_{\mathrm{c}} \mathrm{kg}^{-1}$, COT de 36,4 dag kg-1$^{-1}$ e relação CTC/ COT de 5,8. Entretanto, observou-se que para a LEA 01, entre 45 e 60 dias, e para LEA 02, entre 60 e 90 dias, houve uma redução nas referidas variáveis. Acredita-se que esse fato, bem como as demais variações apresentadas, tanto no Quadro 4 quanto no Quadro 5, deve-se a heterogeneidade do material em compostagem da leira, o que provoca uma degradação e humificação não uniforme do material.

Nos primeiros 30 dias de compostagem do material, no $2^{\circ}$ estágio do processamento, houve acréscimo de $19 \%$ no valor da CTC, juntamente com redução de 12,4 dag $\mathrm{kg}^{-1}$ na concentração de COT. Isto ocorreu devido à degradação da matéria orgânica no reviramento ter sido muito mais rápida que no primeiro estágio, o que proporcionou maior humificação do material, proporcionando aumento no valor da CTC e a transformação do COT em dióxido de carbono, reduzindo, com isso, sua concentração. Além disso, estes valores corroboram, juntamente com a pequena redução na concentração de SV, com o fato de que, no primeiro estágio, tal como conduzido pelos avicultores da Zona da Mata Mineira, praticamente, não ocorre degradação bioquímica do material. Sendo assim, os valores obtidos após os 60 dias podem ser considerados, aproximadamente, os valores que seriam obtidos por balanço de massa dos materiais compostados antes mesmo do início do experimento.

Rodella e Alcarde (1994), misturando serragem e turfa em diferentes proporções, verificaram que a CTC e a relação $\mathrm{CTC/COT} \mathrm{variou}$

Quadro 5. Variável CTC e índice CTC/COT dos materiais compostados.

\begin{tabular}{|c|c|c|c|c|}
\hline Experimentos & Dias & CTC & $\left(\mathrm{cmol}_{\mathrm{c}} \mathrm{kg}^{-1}\right)$ & CTC/COT \\
\hline \multirow{2}{*}{ COMPOSTEIRA } & 60 & & 175 & 4,2 \\
\hline & 90 & & 209 & 5,8 \\
\hline \multirow{3}{*}{ LEA 01} & 45 & & 192 & 5,3 \\
\hline & 60 & & 180 & 5,3 \\
\hline & 90 & & - & - \\
\hline \multirow{4}{*}{ LEA 02} & 30 & & 196 & 5,2 \\
\hline & 45 & & 192 & 5,1 \\
\hline & 60 & & 215 & 7,0 \\
\hline & 90 & & 197 & 5,8 \\
\hline \multirow{4}{*}{ LEA 03} & 30 & & 183 & 4,5 \\
\hline & 45 & & 198 & 5,8 \\
\hline & 60 & & - & - \\
\hline & 90 & & 212 & 9,7 \\
\hline
\end{tabular}


proporcionalmente ao teor de matéria orgânica presente na mistura. Nesse trabalho, a CTC variou de $5,48 \mathrm{cmol}_{\mathrm{c}} \mathrm{kg}^{-1}$, na mistura sem turfa, até 51,57 $\mathrm{cmol}_{\mathrm{c}} \mathrm{kg}^{-1}$, na mistura com $41 \%$ de turfa. A relação CTC/COT variou de 0,35 a 3,38, segundo Harada e Inoko (1980), valores de CTC/COT superiores a 1,7 indicam bom grau de humificação do material orgânico. De acordo com esse valor de referência, os compostos orgânicos obtidos neste trabalho podem ser considerados excelentes compostos orgânicos devido ao seu alto grau de humificação. Nesse sentido, pode ser observada, ao final de cada experimento, a seguinte relação entre o coeficiente de humificação e o valor de referencia $(1,7)$ : composteira (3,5 vezes, nos 30 primeiros dias do $2^{\circ}$ estágio); LEA 01 ( 3 vezes), LEA 02 (4 vezes aos 60 dias, porém reduzindo para 3,4 vezes aos 90 dias, provavelmente em função do ambiente heterogêneo da leira) e a LEA 03 (5,7 vezes aos 90 dias).

Avaliando os índices obtidos nos processos de compostagem que têm, em sua composição, os mesmos materiais, observa-se que: $\mathrm{O}$ material da LEA 03 apresentou coeficiente de maturação, aos 90 dias $(9,7), 1,7$ vezes o valor obtido no material da composteira $(5,25)$. Como pode ser observado no Quadro 4, esse valor foi conseguido não pelo ganho em termos de CTC, mas da redução na concentração de COT. O material da LEA 02 apresentou coeficiente de maturação, aos 90 dias, 1,3 vezes o valor obtido no material da LEA 01 . Essa diferença é devida ao ganho, em termos de CTC, e também à redução na concentração de COT.

Diante destes resultados, pode-se dizer que o índice CTC/COT tenha retratado melhor a evolução do processo de compostagem. Isso, porque enquanto a CTC descreve a redução do tamanho das partículas e consequentemente o aumento da superfície específica, além de estar relacionada ao processo de humificação, o COT está relacionado aos sólidos voláteis ou a matéria orgânica ainda presente no composto. Assim, a sua redução indica também a mineralização do material orgânico em compostagem.

\section{CONCLUSÃO}

- Nas condições avaliadas, a relação $\mathrm{C} / \mathrm{N}$ não se mostrou como parâmetro adequado no monitoramento da compostagem de animais, notadamente carcaças de frango;

- O índice CTC/COT mostrou-se um parâmetro adequado no monitoramento do processo de compostagem de animais, notadamente carcaças de frango. Enquanto a CTC descreve a redução do tamanho das partículas e consequentemente o aumento da superfície específica, além de estar relacionada ao processo de humificação, o COT descreve a presença de matéria orgânica presente e, assim, a sua redução indica a mineralização do material em compostagem.

\section{REFERÊNCIAS}

APHA; AWWA; WEF. Standard Methods for Examination of Water and Wastewater. New York: American Public Health Association, American Water Works Association, Water Environment Federation, 2012. 1946p.

BERNAL, M.P.; ALBURQUERQUE, J.A.; MORAL, R. Composting of animal manures and chemical criteria for compost maturity assessment. A review. Bioresource Technology, v.100, n.22, p.5444-5453. 2009.

BORDER, D. Processes and Plant for Waste Composting and other Aerobic Treatment. Cambridgeshire: U.K. Environment Agency. 2002. 198p. (R\&D Technical Report P1-311/TR)

CHIUMENTI, A.; DA BORSO, F.; RODAR, T.; CHIUMENTI, R. Swine manure composting by means of experimental turning equipment. Waste Management, v.27, n.12, p.1774-1782, 2007.

DAM, A.; NIBBELINK, B.G.; WARD, D. Windrow composting of poultry carcasses. Ontario: Ministry of Agriculture - Food and Rural Affairs. 2009. 8p. (ORDER NO. 09-017, AGDEX $720 / 450)$

DORES-SILVA, P.R.; LANDGRAF, M.D.; REZENDE, M.O.O. Acompanhamento químico da vermicompostagem de lodo de esgoto doméstico. 
Química Nova, v.34, n.6, p.956-961, 2011.

FLYNN, R.P.; WOOD, C.W. Temperature and Chemical Changes During Composting of Broiler Litter. Compost Science \& Utilization, v.4, n.3, p.62-70, 1996.

GOLUEKE, C.G.; LAFRENZ, D.; CHASER, B.; DIAZ, L.F. Composting combined refuse and sewage sludge. Compost science land utilization, v.21, n.5, p.42-48, 1980.

GRAVES, R.E.; HATTEMER, G.M.; STETTLER, D.; KRIDER, J.N.; CHAPMAN, D. Composting. In: (Ed.). UNITED STATES DEPARTAMENT OF AGRICULTURE Part 637 Environmental Engineering - National Engineering Handbook. Washington: NATURAL RESOURCES CONSERVATION SERVICE, 2000. Composting, p. 88

GUARDIA, A.; PETIOT, C.; ROGEAU, D. Influence of aeration rate and biodegradability fractionation on composting kinetics. Waste Management, v.28, n.1, p.73-84, 2008.

HARADA, Y.; INOKO, A. Relationship between cation-exchange capacity and degree of maturity of city refuse composts. Soil Science and Plant Nutrition, v.26, n.3, p.353-362, 1980.

JIMÉNEZ, E.I.; GARCIA, V.P. Composting of domestic refuse and sewage sludge. II. Evolution of carbon and some "humification" indexes. Resources, Conservation and Recycling, v.6, n.3, p.243-257, 1992.

KIEHL, E.J. Fertilizantes organicos. São Paulo: Agronomica Ceres, 1985. 492p.

KUMAR, V.R.S.; SIVAKUMAR, K.; PURUSHOTHAMAN, M.R.; NATARAJAN, A.; AMANULLAH, M.M. Chemical Changes During Composting of Dead Birds With Caged Layer Manure. Journal of Applied Sciences Research, v.3, n.10, p.1100-1104, 2007.

MATOS, A.T.; VIDIGAL, S.M.; SEDIYAMA,
M.A.N.; GARCIA, N.C.P.; RIBEIRO, M.F. Compostagem de alguns resíduos orgânicos utilizando-se águas residuárias da suinocultura como fonte de nitrogênio. Revista Brasileira de Engenharia Agrícola e Ambiental, v.2, n.2, p.199-203, 1998.

MELO, L.C.A.; SILVA, C.A.; DIAS, B.D.O. Caracterização da matriz orgânica de resíduos de origens diversificadas. Revista Brasileira de Ciência do Solo, v.32, n.1, p.101-110, 2008.

MONACO, P.A.V.L.; PAIVA, E.C.R.; MATOS, A.T.D.; FERRES, G.C.; RIBEIRO, I.C.A. Avaliação da relação $\mathrm{C} / \mathrm{N}$ e da qualidade do composto produzido em leiras de compostagem de carcaça e diferentes camas de criatório de frangos. Engenharia na Agricultura, v.21, n.6, p.563-573, 2013.

ORRICO JÚNIOR, M.A.P.; ORRICO, A.C.A.; LUCAS JÚNIOR, J. Compostagem dos resíduos da produção agrícola: cama de frangos e carcaças de aves. Engenharia Agrícola, v.30, n.3, p.538$545,2010$.

PAIVA, E.C.R.; MATOS, A.T.; SARMENTO, A.P.; PAULA, H.M.D.; JUSTINO, E.A. Avaliação de sistema de tratamento de carcaças de frangos pelo método da composteira-windrow. Revista Eletrônica de Engenharia Civil, v.3, n.1, p.19$27,2012$.

PAIVA, E.C.R. Gestão de Resíduos Sólidos Orgânicos - Compostagem: varáveis de projeto e operação. Ed. Novas Edições Acadêmicas. 2014. $160 p$.

PEREIRA NETO, J.T. Manual de compostagem: processo de baixo custo. Viçosa: UFV. 2007. 81p.

RICHARD, T. Estimating Carbon Content. In: The Science and Engineering of Composting. Cornell Waste Management Institute, Cornell University. Disponível em: http://compost.css. cornell.edu/calc/carbon.html. Acessado em: 24/07/2014. 
RODELlA, A.; ALCARDE, J. Avaliação de materiais orgânicos empregados como fertilizantes. Scientia Agrícola, v.51, n.3, p.556-562, 1994.

ROIG, A.; LAX, A.; CEGARRA, J.; COSTA, P.; HERNANDEZ, M.T. Cation Exchange Capacity as a Parameter for Measuring the Humification Degree of Manures. Soil Science, v.146, n.5, p.311-316, 1988.

ROS, M.; GARCÍA, C.; HERNÁNDEZ, T. A full-scale study of treatment of pig slurry by composting: Kinetic changes in chemical and microbial properties. Waste Management, v.26, n. 10, p.1108-1118, 2006.

VALENTE, B.S.; XAVIER, E.G.; MANZKE, N.E.; MORAES, P.D.O.; ROLL, V.F.B. Compostagem da mistura de carcaças de frangos de corte e cama de aviário. Varia Scientia Agrárias, v.2, n.2, p.135-152, 2011.

WANG, K.; LI, W.; GUO, J.; ZOU, J.; LI, Y.; ZHANG, L. Spatial distribution of dynamics characteristic in the intermittent aeration static composting of sewage sludge. Bioresource Technology, v.102, n.9, p.5528-5532, 2011. 